\title{
Competencias directivas para promover la participación: familias en las escuelas básicas
}

\author{
CLAUDIO BARRIENTOS PINEEIRO* \\ PATRICIA SILVA GARCÍA ${ }^{* *}$ \\ SERAFÍN ANTÚNEZ MARCOS ${ }^{* * *}$
}

Universidad de Los Lagos - Chile

Recibido el 11-12-2015; primera evaluación el 04-04-2016; segunda evaluación el 02-05-2016; aceptado el 06-05-2016

\section{Resumen}

El presente artículo aporta conocimiento acerca del estado de la cuestión sobre competencias directivas y dinámicas que circundan el desempeño de los directores de escuelas en torno a la promoción de la participación. Utilizando un enfoque cualitativo y una metodología de estudio de caso se obtuvieron resultados que evidenciaron prácticas y estrategias de promoción tradicionalistas, con implicación pasiva de las familias, con niveles mínimos de participación. El desempeño directivo se presenta restrictivo, con débil presencia de actitudes hacia la empatía, confianza y el hacer dialógico, lo que merma el compromiso de los padres por participar en las escuelas. Se destaca la importancia que se le concede a las habilidades sociales, cualidades personales y valores que deben movilizar los directivos para promover adecuadamente la participación padres y la comunidad.

Palabras clave: dirección escolar, competencias, prácticas, estrategias, participación, liderazgo.

\footnotetext{
Becario del Gobierno de Chile por el Programa de Formación de Capital Humano Avanzando de Conicty, con la obtención de grados de máster y doctor en Ciencias de la Educación por la Universidad de Barcelona. Contacto: clabapineiro@gmail.com

** Profesora lectora de la Universidad de Lleida. Maestra de Educación Primaria, socióloga, máster en Desarrollo Educativo y máster en Gestión y Dirección de Centros Educativos. Es doctora en Pedagogía por la UB. Es profesora en el Departamento de Pedagogía Aplicada de la Universidad Autónoma de Barcelona. Es responsable de la Coordinación Académica del Máster en Gestión y Dirección de Centros Educativos de la UB. Contacto: silva@pip.idl.cat

*** Doctor en Pedagogía, por la Universidad de Barcelona. Actualmente es catedrático de universidad en la Facultad de Pedagogía de la Universidad de Barcelona (Departamento de Didáctica y Organización Educativa). Ha ejercido como: maestro, director escolar, profesor titular de la Escuela Universitaria del Profesorado de la Universidad de Barcelona. Contacto: santunez@iub.edu
} 


\section{Management skills to promote participation: families in basic schools}

\section{Abstract}

This article provides knowledge about the state of affairs on policies and dynamics surrounding the performance skills of school principals regarding the promotion of participation. Using a qualitative approach and methodology Case Study results showed traditionalist practices and promotion strategies, passive involvement of families, with minimum levels of participation were obtained. The management performance is presented restrictive weak presence of attitudes toward empathy, trust and do dialogic, thus weakening the commitment of parents to participate in schools. The importance it attaches to social skills, personal qualities and values that should mobilize managers to adequately promote parent and community participation is emphasized.

Keywords: School management, skills, practices, strategies, participation, leadership.

\section{Competências de gestáo para promover famílias de participaçáo nas escolas básicas}

\section{Resumo}

Este artigo fornece o conhecimento sobre o estado de coisas sobre as políticas e dinâmica que envolve as habilidades de desempenho de diretores de escolas sobre a promoção da participação. Usando uma abordagem qualitativa e metodologia do Estudo de Casos resultados mostraram práticas tradicionalistas e estratégias de promoção, participação passiva de famílias, com níveis mínimos de participação foram obtidos. O desempenho da gestão é apresentada restritiva fraca presença de atitudes em relaçáo à empatia, confiança e fazer dialógica, enfraquecendo assim o compromisso de participação dos pais nas escolas. A importância que atribui às habilidades sociais, qualidades e valores pessoais que devem mobilizar gestores para promover adequadamente pai e participação da comunidade é enfatizada.

Palavras-chave: gestâo escolar, habilidades práticas e estratégias, de participação, de liderança 


\section{INTRODUCCIóN}

La función directiva es una labor cada vez más relevante en la mejora educativa, a la que solo en los últimos años se le ha otorgado una atención preferente por su alto impacto y vinculación con la calidad de los aprendizajes de los estudiantes.

El presente trabajo nos permitió describir, analizar y comprender el papel de los directores ${ }^{1}$ de escuelas básicas completas de Panguipulli en relación con la promoción de la participación de las familias ${ }^{2}$ y la comunidad ${ }^{3}$ en los procesos de mejora, en la que se pudo identificar aquellas competencias profesionales, prácticas y estrategias directivas necesarias para promover procesos de participación, en co-relación con las valoraciones y percepciones que los directores, docentes, asistentes de la educación, familias y representantes de la comunidad tienen de ellas.

Con ese fin, pudimos dar respuesta a interrogantes como: ¿La práctica directiva fomenta o no la participación de las familias y la comunidad en el quehacer educativo? ¿Cómo promueven los directores la participación y qué estrategias utilizan? ¿Cuáles son las principales competencias profesionales que movilizan los directores para promover la participación?, entre otras no menos importantes.

Nos situamos en el papel que desempeñan los directores e indagamos en las prácticas y estrategias directivas que favorecen, o no, la promoción de dicha participación, rescatando la contribución al desarrollo de competencias como elementos favorecedores de una gestión directiva orientadas al impulso de actos formadores para el fortalecimiento de una participación pedagógica de las familias y la comunidad.

\footnotetext{
1 Para facilitar la lectura de este documento, se evita la utilización continuada de la duplicidad de género (director/directora, profesor/profesora, alumnos/alumnas, etc.), entendiéndose que se hace referencia a los dos géneros, sin que ello implique ningún tipo de consideración sexista, ni valoraciones poco respetuosas.

2 Se utilizará este concepto para referirnos de igual manera a los padres o apoderados de los estudiantes. Al hacer alusión a papás se hará referencia al varón.

3 Contexto y territorio cercano a las escuelas compuesto por diferentes organizaciones sociales, tanto comunitarias, gubernamentales y privadas, y el colectivo de habitantes que la integran.
} 


\section{FundAMENTACIÓN TEÓRICA}

La dirección escolar es el pilar fundamental en el desarrollo de las políticas institucionales, razón por la cual el director es el centro principal de la actividad micropolitica en la escuela (Balls, 1994, p. 92), a quien debe considerarse una figura de influencia determinante para establecer el rumbo de su organización. Quienes dirigen, tienen a su haber una serie de prácticas que desarrollar y han de transformarse en el eje de la mejora educativa. Por esto, la dirección escolar y «el estilo de dirección no puede hoy plantearse desde una perspectiva personalista...no es un concepto unívoco, es enormemente complejo y hay que entenderlo y estudiarlo dentro de las distintas culturas en que se desarrolla» (Álvarez, 1996, p. 305).

Su desempeño como líderes debe dinamizar y motivar los procesos de participación de todos los actores educativos, orientando sus actuaciones bajo los principios de corresponsabilidad, cooperación, coordinación y autoridad democrática una participación que debe ser entendida como un acto inclusivo que permita a las personas incidir en el rumbo de la organización a través de sus actuaciones, opiniones y toma de decisiones.

Acercarnos a estos principios exige a los directores ser poseedores de una diversidad de recursos profesionales y personales que guíen sus desempeños. En este sentido, el enfoque competencial surge como una real alternativa, ya que:

[...] si se lleva a cabo con rigor y en profundidad, fomenta que los propios directivos asuman un papel activo en su desarrollo profesional. Se trata de ayudar a adquirir mayor conciencia de su nivel de desempeño en el propio contexto de trabajo, a conocer con mayor profundidad sus puntos fuertes (para explorarlos y aprovecharlos) y, también, sus puntos débiles, con el objeto de plantearse itinerarios de mejora profesional basados en la combinación e integración de diversas modalidades: formación, grupos de autodesarrollo, práctica reflexiva, mentoraje, coaching, etc. (Teixidó, 2008, p. 121).

En atención a los actuales sustentos epistemológicos, ontológicos y las consideraciones hermenéuticas, es pertinente conjugar el fortalecimiento de la gestión directiva a partir de estos lineamientos, por cuanto valoramos su visión holística en relación a los desempeńos laborales y el principio de aprendizaje a lo largo de la vida, lo que le asigna una importancia radical hacia la renovación y adaptación de nuevos cuerpos de conocimiento y modos comportamentales en las funciones directivas (McClelland, 1973; Blas, 2007, Teixidó, 2007, Le Boterf, 2001). 
Entenderemos que una competencia no es solo la suma de dos entidades separadas (trabajador y trabajo), ambos forman una única entidad que correlaciona los atributos del trabajo con la experiencia vivida. Tener consciencia de esta correlación permitirá cimentar las capacidades que abran las puertas al autoaprendizaje y al aprender a partir de esa experiencia, elementos determinantes para desarrollo de competencias.

Abrazamos la mirada del enfoque sociológico o socioconstructivo de las competencias (Rodríguez Moneo, 2011), que centra su atención en el desempeño a partir del componente social, cuyo objetivo se enfoca en determinar las características necesarias de una persona para una actuación exitosa en un grupo social o en una sociedad cambiante, incorporando habilidades sociales, actitudes y valores, recursos mentales y conductas que las personas emplean para comunicarse, aprender, realizar tareas y conseguir un buen desempeño.

Considerando el carácter polisémico del concepto de competencia, muchos autores (Rodríguez Moreno, Serreri \& Del Cimmuto, 2010; Mulder, 2007; Perrenoud, 2012; Bunk, 1994; Levy-Leboyer, 1997; Le Boterf, 2001) hacen acopio de profundas y comunes características en torno a una definición. A partir de las aportaciones de Teixidó (2008b) las concebimos como la «capacidad de identificar recursos (conocimientos, aptitudes, valores, informaciones, rasgos de personalidad, habilidades) ser conscientes de ellos, movilizarlos y ponerlos en acción cuando la situación lo requiere. Se adquiere con el uso integrado de los recursos y se demuestra en el transcurso de la acción en tiempo real» (Teixidó, 2008b).

Una diversidad de modelos y perfiles competenciales (Teixidó, 2008a; Fundación Chile, 2006; Uribe \& Celis, 2012; Mineduc, 2005) resaltan la importancia de las cualidades personales e interpersonales para desplegar una adecuada gestión escolar, considerando los distintos elementos que componen la vida institucional escolar, y no solo en pro de la promoción de la participación. Creemos conveniente ejercer desempeños directivos que abarquen la totalidad de las áreas de gestión de una organización como lo son: académica, administrativa, gobierno institucional, servicios, desarrollo profesional y sistema relacional.

En relación al último de ellos se le concede una connotación muy relevante a estos elementos, ya que constituyen un ámbito de actividad transversal. Agrupan las tareas que se llevan a cabo con el fin de dar respuesta a aspectos propios de las relaciones interpersonales en la regulación de la convivencia, la resolución de los conflictos, la negociación, mecanismos de comunicación, gestión de las emociones, entre otras cualidades necesarias para un perfil directivo que comulga con los lineamientos del desarrollo competencial en favor de la promoción de la participación. 
Para ello será fundamental movilizar competencias relacionales que discurran en las dimensiones intrapersonales (con uno mismo), interpersonales (con los demás), grupales y reticulares (redes sociales y virtuales) (Aramendi, 2008, pp. 144-150) que propendan a una mayor cohesión entre los miembros de la comunidad educativa. En esta línea, ponemos especial consideración a las competencias emocionales propuestas por Goleman (1996, pp. 75-76) donde los directores focalicen su atención en el conocimiento de las propias emociones, capacidad para controlar, motivarse a sí mismo, reconocimiento de las emociones ajenas y el control de las relaciones.

En concordancia con los ejes centrales de estudio es dable señalar que el desarrollo de competencias con foco preferente en los aspectos antes mencionados permitirá el fortalecimiento de un espontáneo liderazgo, el que precisa ser gestionado de manera sostenible y compartida con los miembros de la comunidad educativa ${ }^{4}$, a razón de que «mucho de lo que conocemos hoy en el ámbito de la dirección se fundamenta en la investigación sobre liderazgo escolar, cuyo resultado es expresión del impacto que el líder es potencialmente capaz de provocar en las prácticas profesionales docentes, la convivencia y las relaciones con la comunidad» (Uribe y Celis, 2012, p. 113).

\section{Marco metodológico}

La investigación se desarrolló a través de un enfoque cualitativo, desde un paradigma interpretativo, con un diseño etnográfico que se focalizó en «describir y analizar ideas, creencias, significados, conocimientos y prácticas de grupos, culturas y comunidades» (Patton, 2002 citado en Hernández, Fernández \& Baptista, 2006, p. 697), que centró su atención en un contexto social multidimensional y natural, cuyo objeto de estudio fue analizado en su dinámica normal, sin manipularlo ni modificarlo. Rodríguez Gómez, Gil Flores y García Jiménez (1999, p. 32) avalan nuestro enfoque al manifestar que una investigación cualitativa "estudia la realidad en su contexto natural, tal y como sucede, intentando sacar sentido de, o interpretar los fenómenos de acuerdo con los significados que tienen para las personas implicadas». Desde una perspectiva fenomenológica realizamos comprensiones inductivas mirando el escenario de estudio de manera holística e interactuando con los informantes de manera natural y no intrusiva.

\footnotetext{
4 Para el caso del presente estudio nos referiremos a ella entendiendo que dicha comunidad la componen los docentes, el personal no docente — también llamados asistentes de la educación—, los estudiantes y las familias.
} 
De acuerdo a ello, Ruíz Olabuénaga (2009, p. 26) sostiene que los métodos cualitativos estudian significados intersubjetivos, situados y construidos; eligen la entrevista abierta y la observación directa; estudian la vida social en su mismo marco natural sin distorsionarla ni someterla a controles experimentales y; eligen la descripción espesa y los conceptos comprensivos del lenguaje simbólico.

Con estas orientaciones pudimos comprender la realidad del comportamiento particular e idiosincrásico de los participantes, conociendo sus opiniones, actitudes y razones por la cuales se desenvuelven de una determinada manera en este espacio sociocultural que les es propio.

Nos focalizamos en un estudio de caso (Stake, 2007, p. 16), ya que siguiendo las orientaciones de Latorre, Del Rincón y Arnal (1996, p. 233) y Sandín (2010, p. 174), este «constituye un método de investigación para el análisis de la realidad social de gran importancia en el desarrollo de las ciencias sociales y humanas y representa la forma más pertinente y natural de las investigaciones orientadas desde una perspectiva cualitativa».

\section{a. Recogida de la información}

Nos sirvieron como fuente principales de información diez directores de escuelas, con quienes realizamos entrevistas semiestructuradas individuales. Las fuentes complementarias la constituyeron grupos representativos promedio de seis docentes, asistentes de la educación, padres representantes de centros generales de padres ${ }^{5}$ y de la comunidad, con quienes indistintamente realizamos cuatro grupos de discusión.

De igual manera, desarrollamos observaciones no participantes con cinco de los diez directores, con quienes estuvimos en cinco reuniones con padres, tres de actividades de participación en sus escuelas y en diez en jornadas de un día de trabajo con ellos. Finalmente, aplicamos un cuestionario mixto (cualitativo y cuantitativo) a una muestra de 168 padres y apoderados.

\section{b. Análisis de la información}

La información reunida en las entrevistas y grupos de discusión fue analizada inductivamente utilizando el programa informático ATLAS.ti 7 y se consideraron cuatro niveles de análisis: fragmentación de la información, codificación, categorización y obtención de metacategorías.

5 En adelante centro general de padres. 
Los focos temáticos, principios, criterios y códigos utilizados fueron los mismos en ambas estrategias, debido al carácter transversal de la información. Esto permitió realizar una triangulación ordenada y coherente para la obtención de los resultados.

Del mencionado proceso emergieron las principales metacategorías que dan respuesta a las inquietudes investigativas, las que se muestran en la tabla 1.

\section{Tabla 1. Metacategorías y definiciones}

\begin{tabular}{|l|l|l|}
\hline Código & Metacategorías & Definición \\
\hline CP & $\begin{array}{l}\text { Competencias } \\
\text { profesionales }\end{array}$ & $\begin{array}{l}\text { Conjunto combinado de saberes, habilidades, destrezas, } \\
\text { actitudes, valores, motivaciones y rasgos de personalidad } \\
\text { que articulan y movilizan los directores en sus prácticas } \\
\text { directivas para la efectiva promoción de la participación } \\
\text { de los padres y la comunidad en sus escuela. }\end{array}$ \\
\hline PD & $\begin{array}{l}\text { Prácticas } \\
\text { directivas }\end{array}$ & $\begin{array}{l}\text { Conjunto de comportamientos orientados a la concreción } \\
\text { de la promoción de la participación de las familias y la } \\
\text { comunidad en la escuela. }\end{array}$ \\
\hline EPP & $\begin{array}{l}\text { Estrategias de } \\
\text { promoción de la } \\
\text { participación }\end{array}$ & $\begin{array}{l}\text { Conjunto de acciones planificadas y concretas en que los } \\
\text { directores desentrañan sus modos de hacer con el pro- } \\
\text { pósito de promover la participación de las familias y la } \\
\text { comunidad, movilizando una variedad de recursos, dispo- } \\
\text { sitivos, herramientas y decisiones para ello. }\end{array}$ \\
\hline VAE & $\begin{array}{l}\text { Visiones de los } \\
\text { agentes edu- } \\
\text { cativos y la } \\
\text { comunidad }\end{array}$ & $\begin{array}{l}\text { Consideraciones, percepciones y valoraciones de los docen- } \\
\text { tes, padres, asistentes de la educación y la comunidad en } \\
\text { relación a las competencias profesionales y desempeńo de } \\
\text { los directores en relación a la promoción de la participa- } \\
\text { ción de las familias y la comunidad en las escuelas. }\end{array}$ \\
\hline
\end{tabular}

Con relación a la información de los cuestionarios de padres esta se acopió en la aplicación google form, desde donde se realizó un análisis manual cualitativo de las respuestas abiertas y considerando las categorías y metacategorías obtenidas del análisis anterior. La información cuantitativa fue tabulada y graficada por la misma aplicación y permitió contextualizar y conocer las principales características de la muestra y las apreciaciones y significados que los padres le atribuyen a los demás objetivos del estudio. estas fueron interpretadas cualitativamente sustrayendo su referencia cuantitativa, lo que permitió obtener mayor claridad al momento de la obtención de los resultados. 
En relación a la observación no participante el proceso se realizó de manera manual, organizada por áreas temáticas distribuidas a partir de los objetivos específicos del estudio y perseguían complementar y entramar el discurso de los directivos en torno a las dinámicas de sus relaciones sociales y las competencias que movilizan hacía la promoción de la participación.

\section{Resultados}

Posterior al proceso de análisis los principales resultados obtenidos se relacionan con que:

Los directores le conceden un papel fundamental a las cualidades personales, cimentadas en valores humanos, para promover la participación. Consideran relevante movilizar habilidades sociales como elementos esenciales para sostener adecuados niveles de relaciones interpersonales con los agentes educativos. De manera unánime asignan una importancia complementaria a los conocimientos teóricos propios de sus cargos para la eficiencia de su trabajo.

En relación con las cualidades personales, entendidas como características propias de un individuo que refleja su manera de ser, manifestaron que en general son personas cordiales y humildes. Un ejemplo de ello es: «Mi línea es del trato cordial, el saludo afectuoso, el preguntarle por su persona». ED10J. rtf - 1:6 (9:9). De igual modo dijeron ser personas creíbles y consecuentes, razones por la cual sienten que con ello logran generar mayor confianza con los padres.

Respecto de las habilidades sociales, entendidas como conductas aprendidas que se manifiestan en situaciones interpersonales y que nos permiten interactuar con las personas de manera adecuada, consideran que son competencias fundamentales para promover la participación. Declararon que las más representativas tienen que ver con las habilidades comunicacionales. Dijeron movilizar la empatía, el diálogo y la escucha. De acuerdo a esto han manifestado que: "Un directivo tiene que escuchar, tiene que hablar, tiene que entender y ponerse en el lugar del otro». ED4D.rtf - 5:33 (19:19).

Consideran que quienes ocupen un cargo directivo deben ser humildes, condición que debe primar por sobre la autoridad conferida por el cargo, idea que también es compartida por todos los agentes educativos. De acuerdo a esto afirman que: «Yo creo que los directores debemos ser humildes en ostentar el cargo, porque el ser así no significa que uno sea menos, la humildad no debe confundirse con ineficiencia y debilidad de liderazgo». ED7G.rtf - 8:57 (16:16). 
Sin embargo, las indagaciones realizadas con los padres, y otros agentes educativos, evidencian una percepción contrapuesta. Los padres, ante la pregunta del cuestionario ¿Cuáles son las características profesionales o personales de los directores que consideran fundamentales para promover la participación? hacen sentir la importancia que le asignan a las cualidades personales y a las habilidades sociales. Un $73 \%$ es enfático en señalar que los directivos deben ser más cercanos y asequibles a los padres, además de mantener una buena comunicación con ellos. Agregan que es fundamental que sean personas comprensivas, empáticas, sociables y con capacidad de escucha, condiciones que, en menor o mayor grado, echan en falta en los directivos de la comuna.

Los resultados parecen mostrar que los directores asumen su labor con un cierto dejo de autoridad que disocia la cohesión relacional y los grados de confianza con los padres, toda vez que en ello se juegan sus sustentos la empatía, la escucha activa, la humildad, la crítica y la autocrítica, condiciones imposibles de gestionar si no existe una adecuada relación dialógica. Parece ser que lo que manifiestan transmitir los directores hacia los padres no es visualizado de la misma manera por ellos, lo que nos inclina a pensar que con la mayoría de los padres se presenta una relación distante que no les permite verse.

Con relación a los valores, entendidos como conceptos universales de comportamiento social y éticamente aceptados, los directores resaltan el respeto, la solidaridad, la honestidad y la responsabilidad como atributos que guían su desempeño diario. Afirmaciones como estas sostienen sus convicciones: Los trato como personas. Soy muy respetuosa con los padres y de sus puntos de vista. ED10J.rtf - 1:63 (19:19).

En términos valóricos no se evidencian grandes discrepancias. Sin embargo, las afirmaciones de los padres — a través del grupo de discusión y el cuestionario- dejan entrever ciertas diferencias en torno a la humildad y a los grados de relación de confianza que se generan con los directores. Ante la pregunta en el cuestionario de padres ¿Qué deberían hacer los directores para lograr una mayor participación de los padres en la escuela?, un porcentaje superior al $60 \%$ se refirió a esto graficándolo de esta manera: «Que genere confianza, en este caso, hacia los apoderados, que los apoderados sintamos la confianza de ir a conversar con él» GD-A.rtf - 1:136 (62:62). Es pertinente decir que dichos niveles de confianza son más estrechos entre los directores y la minoría de padres que participa activamente en las directivas de los sub-centros y el CGP, debido a la cercanía que se produce por los compromisos de sus cargos.

$\mathrm{Al}$ igual que los padres, docentes y asistentes de la educación, los directores identifican en el liderazgo una competencia fundamental para el éxito de su 
desempeño en relación con el tema de estudio y para su desempeño profesional general, lo que se acerca a la visión de Leithwood (2009); Leithwood, Harris y Hopkins (2008) y; Harris y Chapman (2002); Álvarez (2010). Todos reconocen poner en juego un liderazgo democrático, compartido y participativo. Sin embargo, el análisis de la información nos hace sostener que al parecer dicho liderazgo no se presenta definido. A través de las observaciones realizadas en el campo pudimos evidenciar que existe el conocimiento acerca del ejercicio de un liderazgo democrático y compartido, pero que paradójicamente no se deja ver claramente en la promoción de la participación con los padres, aunque sí en lo laboral con los docentes y los asistentes de la educación.

- Competenciales por fortalecer

Los relatos de los directores develan que la tan necesaria humildad no se manifiesta en la práctica como se quisiera. Al menos seis de los directivos creen que sus colegas se apropian inadecuadamente de la calidad de autoridad. No obstante, ninguno de ellos lo asume como una falencia propia, sino que la observan en los otros. La presente afirmación aclara dicho planteamiento: "Yo creo que a los directores nos falta apertura personal, creo que los directores abusan mucho del concepto de autoridad». ED7G.rtf - 8:75 (16:16).

Sin embargo, la apreciación por parte de los integrantes de los otros estamentos es menos crítica. Los asistentes de la educación y los docentes creen que dicha condición está en vía de extinción y que hoy en día aquello no ostenta tanta notoriedad. Coinciden en que los nuevos directores - que han asumido sus cargos en los últimos años- son personas más abiertas, sencillas, cercanas y que poseen un liderazgo pedagógico más encausado. En relación a esto afirman que: «Yo creo que se está instalando eso. Los directores que han asumido entre el año pasado y este traen cosas como estas, sí». GD-D. $\mathrm{rtf}-4: 40$ (32:32).

Por su parte, los agentes comunitarios creen que las competencias profesionales y personales las observan con un desequilibrio muy dispar. Ellos no visualizan un liderazgo claro, democrático y participativo. Observan que los directores, en su mayoría, carecen de un posicionamiento de opinión y acción frente a determinados temas de contingencia social (políticas, ambientales, sindicales, entre otros), manteniendo un perfil excluyente que no se condice con una figura de líderes comunitarios que debieran tener y con el discurso de la corresponsabilidad educativa que han manifestado. Declaran que los directores se limitan a hacer y decir lo que les corresponde solo en relación con su cargo y funciones. 
Una afirmación al respecto dice que un director: «Debe ser una persona afable, cercana, conciliadora, que participe y se integre en la vida de la comunidad y el entorno. Es importante que él esté en los sucesos importantes. Más allá de su figura de autoridad, deben tener un sentido vinculante con la comunidad...» GD-C.rtf - 3:38 (37:37).

Por otro lado, los directores manifiestan precariedad en ciertos conocimientos y habilidades. Coinciden en que deben permitirse delegar más funciones y responsabilidades en los funcionarios de sus escuelas como también en los padres, ya que reconocen que es una complicación para ellos que los asuntos que delegan se realicen en tiempos y de manera distinta a como ellos lo harían. Un ejemplo de aquello es: «Yo quiero que todas las personas funcionen como funciono yo y creo que ese es un problema de los directores en general, que yo quiero que las cosas se hagan como yo las hago y a la velocidad que uno quiere». ED6F.rtf - 7:115 (33:33).

Igualmente debilitado, consideran al menos seis de los directores, son los pocos conocimientos y experiencia directiva que poseen. Sienten que solo el bagaje profesional les permitirá desarrollar aquellas competencias que les pudieran estar limitando su desempeño. De esto da cuenta la siguiente afirmación: «Me falta mucho bagaje de conocimientos, de la práctica en sí, de la experiencia, me falta todavía mucho por desarrollar, por crecer, por conocer». ED1A.rtf - 14:196 (40:40).

- Prácticas y estrategias directivas

Concebidas las prácticas directivas como el conjunto de comportamientos que reflejan aquello que los directores hacen en relación a nuestro estudio; y las estrategias directivas como el conjunto de acciones planificadas y concretas que desentrañan los modos de hacer, podemos decir que:

En relación a las prácticas señalan que una de las más tradicionales es la entrega permanente de información a las familias sobre el acontecer de la escuela y el rendimiento educativo de los estudiantes. La estrategia más utilizada para ello son las reuniones de padres y la entrega de información a la comunidad a través de los medios de comunicación local escritos y orales, con el objeto de resaltar y difundir principalmente los logros institucionales.

En relación a la toma de decisiones, al menos nueve directivos afirman que dentro de sus prácticas habituales se encuentra considerar las opiniones de los padres y tomar decisiones compartidas con ellos, principalmente con las directivas del CGP y los representantes de los subcentros. La mayoría de los padres (72\%) ratifican esto mismo y declaran estar de acuerdo y totalmente de acuerdo con ello. Su percepción, en este sentido, es bastante positiva, 
lo que parece evidenciar que los directores están abiertos y dispuestos a escucharlos. Sin embargo, es conveniente precisar que no fue posible establecer qué nivel de consideración le asignan efectivamente estos a la mencionada estrategia.

Se estableció que no es habitual realizar consultas masivas que permitan conocer las opiniones, ideas o aportaciones de la totalidad de los padres sobre temas relevantes. La toma de decisiones en general se realiza en reuniones con las directivas del CGP o con los delegados de los subcentros de cursos. Esta forma de participación pudiéramos considerarla reduccionista, tanto por su cobertura como también por las limitadas áreas de injerencia, donde lo pedagógico, didáctico y curricular no tienen cabida.

En otro ámbito, los directores manifestaron — con distintos grados de relevancia- que consideran en sus planificaciones estrategias sobre aspectos logísticos y brindar condiciones óptimas para las convocatorias. Casi todos quienes cuentan con padres que viven lejos de las escuelas se preocupan que en las reuniones puedan ofrecer, dentro de las posibilidades, una merienda y que los horarios para las mismas sean adecuados al transporte público y a la disponibilidad de tiempo de los padres.

Estos mismos, al ser consultados acerca del grado de satisfacción con el papel que desempeñan los directores en la promoción de la participación un $68 \%$ declaró estar muy satisfecho $(38 \%)$ y bastante satisfecho $(30 \%)$, lo que representa un resultado suficientemente considerable de satisfacción.

En consideración a la baja asistencia de los padres a las reuniones, la mayoría de los directores manifestaron que están impulsando un cambio de estrategia paulatina en la estructura temática de las mismas. Era habitual en ellas planificar beneficios y convivencias, ante lo cual solían solicitar aportes en dinero o trabajo, situación que terminó por agobiarlos, lo que podría explicar una razón sobre el bajo interés de los padres por asistir.

En el área comunitaria, los directores tienen gran consideración por promover la participación de redes de apoyo como soporte de mejora educativa. $\mathrm{Al}$ menos ocho de ellos afirmaron mantener una adecuada comunicación con ellas. Estratégicamente esto lo realizan enviando invitaciones e informando del acontecer de la escuela. Acerca de esto nos dicen que lo hacen: "Invitándolos a venir a la escuela o entregándoles información a la gente e instituciones que están más distantes y que constituyen nuestras redes de apoyo. Eso lo hacemos y nos ha dado estupendos resultados». ED8H.rtf - 9:171 (10:10). De igual manera lo hacen a través del teléfono, con algunas visitas o por medio del correo electrónico. 
Nueve de los diez directores, a excepción de una escuela rural con una dispersión geográfica muy alta de familias, facilita los espacios físicos para el uso de las instituciones de la comunidad. De igual forma ponen a disposición los recursos para llevar a cabo iniciativas educativas de organizaciones gubernamentales y privadas.

En esta misma línea, un 74\% de los padres está de acuerdo y totalmente de acuerdo ( $40 \%$ y $34 \%$ respectivamente) en que los directivos estimulan y facilitan la participación de personas y organismos externos a las escuelas con el objeto de enriquecer la calidad del servicio educativo de sus escuelas.

Una práctica muy habitual y concreta es promover la asistencia y participación de los padres, redes de apoyo y sus comunidades en los variados eventos, ceremonias y celebraciones que se realizan durante el ańo escolar, ya sean como invitados observadores, colaboradores o participantes activos, aunque lo predominante es ser observadores pasivos.

Una de las prácticas más relevantes, que pone el techo a las anteriores y da sentido al presente estudio, tiene que ver con que si los directores promueven o no la participación de las familias y la comunidad en sus escuelas. En este escenario, docentes, asistentes de la educación y padres coindicen en señalar que sí lo hacen y que las escuelas realizan esfuerzos necesarios para que ello ocurra. Lo anterior lo afirman manifestando que: «Sí (unánime). Sí, nosotros creemos que las escuelas en general promueven la participación». GD-A.rtf 1:37 (29:29).

Por último, una práctica no generalizada y que efectivamente podría repercutir positivamente en la promoción de la participación es dotar de mayor autonomía al trabajo a los CGP para que realicen sus propias reuniones y propongan o planifiquen actividades en coordinación con las escuelas. Parece ser que las motivaciones para que ello ocurra no nacen ni se priorizan desde ambos sectores. Al respecto solo tres de ellos se manifestaron en este sentido. $\mathrm{Al}$ respecto uno de ellos señaló que: «Yo le doy libertad al Centro de Padres para que ellos trabajen y decidan en que aportar a la escuela». ED4D.rtf - 5:95 (13:13).

\section{Conclusiones}

Cada vez más se reconoce a la dirección escolar como una función altamente relevante dentro del sistema educativo. Concluimos que se debe seguir profundizando en estudios relacionados con este desempeńo y atender a su desarrollo profesional, toda vez que si bien los directores promueven una participación, esta se sustenta en un activismo de presencia superficial y festiva, con reducido nivel de incidencia de las familias en aspectos educativos. 
Valoramos la importancia del papel directivo en la promoción de la participación de las familias y la comunidad. Como líderes educativos deben hacer verbo convicciones conceptuales tan importantes como el sentido compartido del acto de educar, de comunidad y de participación pedagógica. Solo así lo educativo dejará de reducirse solo al espacio escolar y se abrirá a la comunidad, permitiendo a las escuelas continuar con la tarea educativa iniciada en las familias y en la comunidad.

La movilización de competencias directivas admite un desempeño fundado en elementos personales e interpersonales que consideramos indispensable para promover la participación. A través de ellas será posible cimentar una gestión más cercana y humanizadora, en conjunción con conocimientos técnicos y teóricos suficientes. Estas competencias deben vincularse directamente con habilidades sociales, cualidades personales, interpersonales y valores que deben ser puestos en acción por los directores para promover la participación.

Cualidades personales y valores como la cordialidad, la humildad, la empatía y el diálogo son indispensables en la promoción de la participación y deben ser cultivados permanentemente por los directores. Es indispensable desvincular la omnipresente autoridad heredada del cargo. Una gestión directiva humanizadora, dialógica y cercana permitirán eliminar aquellas barreras que afianzan las divergencias entre las escuelas y los padres.

Una educación participativa requiere de directores que movilicen competencias y estrategias que otorguen mayor cobertura de autonomía y autogestión a los padres para tomar decisiones en aspectos relevantes para el quehacer institucional, incluido lo curricular, dentro de las posibilidades que entrega el contexto sociocultural. Resulta paradójico que teniendo las facultades para hacerlo los directivos no se lo permitan.

Los directores deben reconocerse como detentores de un liderazgo pedagógico visible, que a la luz de un liderazgo compartido permitan que los agentes educativos puedan validarse y aportar sus potencialidades al proceso educativo. Con una idea estrecha de lo que es la educación se retrotrae lo educativo al conocimiento disciplinar y la participación de las familias y la comunidad se torna funcionalista y desintegradora.

La dirección escolar debe fundar sus funciones en dos dimensiones relevantes:

- Gestionar todos los ámbitos de la actividad institucional (académico, administrativo, gobierno institucional, servicios, desarrollo profesional, sistema relacional) bajo principios de actos formadores, entendidos como prácticas de gestión que focalizan su atención en la formación de los estudiantes, 
vinculados a un sentido educativo sobre lo que se proyecta que se espera de las niñas y los nińos, actos que deben trascender la sola ejecución burocrático-administrativa de su desempeño y lo meramente disciplinar.

- Promover una cultura de participación pedagógica de las familias y la comunidad, que fortalezca su implicación con un sentido formativo, más allá de lo estrictamente curricular.

Concebida de esta manera, una verdadera participación pedagógica dotará de sentido a aquellos actos formadores, lo que marcará la diferencia entre un administrador educacional y un director «educador».

Los procesos de participación en las escuelas son complejos y su promoción no depende exclusivamente de las competencias directivas, aunque sí es un elemento decidor en su promoción y fortalecimiento. Por esto será conveniente cimentar una dirección escolar bajo un arraigado liderazgo compartido y sostenible, que reconozca y homologue el potencial liderazgo pedagógico que pueden ostentar las familias y la comunidad en su conjunto. La delegación de funciones no debe ser un acto restrictivo.

\section{REFERENCIAS BIBLIOGRÁFICAS}

Álvarez, M. (1996). Los estilos de dirección y sus consecuencias. Bases para su configuración como estrategia de intervención. En G. Domínguez Fernández y J. Mesanza (Coords.), Manual de organización de instituciones educativas (pp. 303-343). Madrid: Escuela Española.

Álvarez, M. (2010). Liderazgo compartido. Buenas prácticas de dirección escolar. Madrid: Grefol.

Aramendi, P. (2008). Las competencias relacionales en la dirección. En J. Gairín, J. y S. Antúnez (Coords.), Organizaciones educativas al servicio de la sociedad. Barcelona: Wolters Kluwer.

Balls, S.J. (1994). La Micropolítica de la escuela. Hacia una teoría de la organización escolar. Barcelona: Paidós.

Blas, F. (2007). Competencias profesionales en la formación profesional. Madrid: Alianza.

Bunk, G. (1994). La transmisión de las competencias en la formación y perfeccionamiento profesional de la RFA. Revista europea de formación profesional, 1, pp. 8-14. Recuperado de http://goo.gl/FLpnts

Fundación Chile (2006). Perfiles de Competencias Directivas, Docentes y Profesionales de apoyo. Programa de Educación, Gestión Escolar. Fundación Chile. Recuperado de http://goo.gl/pMWq3E 
Goleman, D. (1996). Inteligencia emocional. Barcelona: Kairós.

Harris, A. \& Chapman, C. (2002). Democratic leadership for school improvement in challenging contexts. International Electronic Journal for Leadership in Learning, 6(9). Recuperado de http://goo.gl/jP1mFX

Hernández, R., Fernández, C. \& Baptista, P. (2006). Metodología de la investigación. México: Mc Graw Hill.

Latorre, A., Del Rincón, D. \& Arnal, J. (1996). Bases metodológicas de la investigación educativa. Barcelona: GR92.

Le Boterf, G. (2001). Ingeniería de las competencias. Barcelona: Epise.

Leithwood, K. (2009). ¿Cómo liderar nuestras escuelas? Aportes desde la investigación. Santiago de Chile: Área de Educación, Fundación Chile.

Leithwood, K., Harris, A. \& Hopkins, D. (2008). Seven strong claims about successful school leadership. School Leadership and Management, 28(1), 27-42. https://doi.org/ 10.1080/13632430701800060

Levy-Levoyer, C. (1997). Gestión de las competencias: como analizarlas, como evaluarlas, como desarrollarlas. Barcelona: Ediciones Gestión 2000.

McClelland, D.C. (1973). Testing for Competencies rather than intelligence. American Psychologist, 28, 1-14. https://doi.org/10.1037/h0034092

Mineduc (2005). Marco para la Buena Dirección. Criterios para el desarrollo profesional y evaluación del desempeño. Recuperado de http://goo.gl/Tc0PZ7

Mulder, M. (2007). Competencias. La esencia y utilización del concepto en la formación profesional inicial y permanente. Revista europea de formación profesional, 40(1), pp. 5-24. Recuperado de http://goo.gl/dV63OQ

Perrenoud, P. (2012). Cuando al escuela pretende preparar para la vida ¿Desarrollar competencias o preparar para la vida?. Barcelona: Graó.

Rodríguez Gómez, G., Gil Flores, J. \& García Jiménez, E. (1999). Metodología de la investigación cualitativa. Málaga: Aljibe.

Rodríguez Moneo, M. (2011). El proceso de enseñanza y aprendizaje de competencias. En F. Labrador y R. Santero (Eds.), Evaluación global de los resultados del aprendizaje en las titulaciones dentro del Espacio Europeo de Educación Superior. Madrid, España: Dykinson. Recuperado de http:// goo.gl/jilOhl

Rodríguez Moreno, M.L., Serreri, P. \& Del Cimmuto, A. (2010). Desarrollo de competencias: Teoría y práctica. Balance, proyecto profesional y aprendizaje basado en el trabajo. Barcelona: Laertes

Ruíz Olabuénaga, J. (2009). Metodología de la investigación cualitativa. Bilbao: Universidad de Deusto. 
Sandín, M. P. (2010). La investigación cualitativa en educación. Fundamentos y tradiciones. Madrid: McGraw Hill.

Stake, R. (2007). Investigación con estudio de casos. Madrid: Morata.

Teixidó, J. (2007). Competencias para el ejercicio de la dirección escolar. XVIII Jornadas Estatales del Fórum Europeo de Administradores en Educación. Recuperado de http://goo.gl/HVyMKT

Teixidó, J. (2008a). Competencias profesionales de los directivos escolares. Identificación y desarrollo. En J. Gairín y S. Antúnez (Eds.), Las organizaciones educativas al servicio de sociedad (pp. 121-135). Madrid, España: Wolters Kluwer.

Teixidó, J. (2008b). Competencias profesionales de los directivos escolares. Grupo de investigación en organización de centros. Recuperado de http://goo. gl/VRdqfz

Uribe, M. \& Celis, M. (2012). Desarrollo de capacidades para un liderazgo escolar efectivo. En J. Weinstein y G. Muńoz (Eds.), ¿Qué sabemos sobre los directores de escuelas en Chile? (pp. 113-132). Santiago de Chile: Salesianos impresores. 